\title{
Prediction of Interbank Offered Rate Based on Time Series Model
}

\author{
Ding Huang ${ }^{1 *}$, Ming Zhong ${ }^{2}$ and Xupeng Shi $^{3}$ \\ ${ }^{123}$ School of Software Engineering, Chongqing University of Posts and Telecommunications, Chongqing, 400065, China
}

\begin{abstract}
This paper studies the prediction of interbank offered rate changes in each working day. Using the actual data of each working day of China's interbank offered rate from 2007 to 2019, this paper sets up ARIMA, Prophet, grey model and MTGNN to study and verify the time series data, and make a comparison between these models. The limitation of this paper is that it does not consider the impact of macroeconomic characteristics but only considers the predict changes in time series. The results of this paper are expected to be helpful for bank management and interbank transaction decision making.
\end{abstract}

\section{Introduction}

Time series data is usually one series or multiple series of numerical data represented in a time dimension. In real life, it is a common activity to record the changes of these time series data, including a large number of time series data in the fields of weather, commerce, agriculture, finance and medical research. In short, the prediction research for time series data not only covers many application fields, but also plays an important role in these fields. As the benchmark of financial product pricing, the term structure of interest rate can not only provide guarantee for the country to formulate scientific and reasonable macro policy, but also provide support for China's financial product pricing and risk management, and promote the stable development of macro economy and the process of interest rate marketization. Therefore, the research on the interbank interest rate prediction can not only help the national economic market to understand the accurate real-time economic trend, but also provide the basic reference for the financial institutions such as banks to regulate the market and the trend of the economic situation ${ }^{1}$.

The work of this paper is as follows. The second chapter introduces the related work of time series data prediction. The third chapter introduces the prediction method adopted in this paper. The fourth chapter is the experiment of interbank offered rate prediction. The fifth chapter summarizes the research work and conclusion of this paper.

\section{Related work}

The prediction of interbank offered rate has gradually become one of the research directions of time series prediction. By using time series model or machine learning model to analyze and train the change rule of historical time series data of interbank offered rate, we can further predict the trend of future data by learning the fluctuation and change of historical data ${ }^{2}$.

Modarres $^{3}$ used the seasonal autoregressive integrated moving average (SARIMA) linear model to predict the monthly flow of a river in Iran and proved the SARIMA model in predicting the usefulness of water resources planning and management. Junior ${ }^{4}$ et al. used ARIMA model to evaluate the performance of time series prediction of Brazil stock market index and proved that ARIMA model could be used for time series indexes related to stock market index prediction. Gorr ${ }^{5}$ et al compared the prediction accuracy of the univariate time series model with the commonly used naive method, proving that the Holt index smoothing and monthly seasonal use of the data estimation of a city in the United States are the most accurate models for predicting regional crime series. $\mathrm{Nepal}^{6}$ et al. proposed a hybrid model including clustering and ARIMA to predict the power load of a university building. It has been proved that the combination of clustering and ARIMA model can improve the prediction performance.

In order to further improve the prediction accuracy, researchers at home and abroad introduced the machine learning algorithm for time series prediction. Dash ${ }^{7}$ et al. used the fuzzy neural network (FNN) to predict the time series of power load. In the supervised learning stage, the Kalman filter update equation of the fuzzy neural network was given, and in the supervised learning stage, the convergence and precision of the fuzzy neural network were better than that of the gradient descent back propagation algorithm. $\mathrm{Liu}^{8}$ et al. in order to explore the multifrequency stock model, this paper proposes a model adaptive wavelet transform (AWTM), according to the dynamic changes of the input sequence automatically focus on different frequency component in order to solve the problem of the stock prediction. Alves ${ }^{9}$ et al used random forest regressors to predict crime and quantify the influence of urban indicators on homicide. The results confirmed the importance of urban indicators on crime

\footnotetext{
*Corresponding author's e-mail: s181231012@stu.cqupt.edu.cn
} 
prediction and revealed that unemployment and illiteracy were the two most important factors affecting homicide.

In the studies of the above two types of time series prediction methods, most of the time scales of time series data are in months or even years. For the daily data of the interbank offered rate, it is more important to be able to predict the continuous value of the offered rate, so as to ensure the stability of its change trend.

\section{Method introduction}

\subsection{Prophet}

Prophet ${ }^{10}$ is a time series prediction algorithm open source by Facebook. In Prophet's algorithm, these three items are also split and fitted to complete the prediction. Prophet model adopts the generalized addition model to fit and forecast the time series, and its combination formula is:

$$
y(t)=g(t)+s(t)+h(t)+\varepsilon_{t}
$$

Where $g(t)$ is the fitting function of the piecewise linear growth or logical growth in the time series, and its expression is as follows:

$$
g(t)=\frac{C}{1+e^{-k(t-m)}}
$$

When the trend of this part grows linearly, the trend term will degenerate into a linear function, as shown below:

$$
g(t)=\left(k+a(t)^{T} \delta\right) t+\left(m+a(t)^{T} \gamma\right)
$$

$s(t)$ stands for periodic change, which is taken into account in almost all time series. Therefore, the function of the season term is expressed by Fourier series, as follows:

$$
s(t)=\sum_{n=1}^{N}\left(a_{n} \cos \left(\frac{2 \pi n t}{T}\right)+b_{n} \sin \left(\frac{2 \pi n t}{T}\right)\right)
$$

Where $T$ represents the period, and $N$ is set according to different periods. $h(t)$ is the irregular holiday effect. In order to represent the influence of different holidays, a corresponding indicator function is needed, and a parameter $\mathrm{k}$ is needed to represent the influence range of holidays, as shown below:

$$
\begin{gathered}
h(t)=Z(t) \\
Z(t)=\left[1\left(t \in D_{1}\right), \ldots, 1\left(t \in D_{L}\right)\right] \\
k \in \operatorname{Normal}\left(0, v^{2}\right)
\end{gathered}
$$

Where let each holiday be $i$, let $D_{\mathrm{i}}$ be the set of the past and future time list of the i-th holiday, and $v$ is the influence of the holiday. The greater $v$ is, the greater the influence of the holiday will be. In the formula (4), $\varepsilon_{t}$ represents the error term, which can also be called the residual term. Prophet algorithm is to get the predicted value of time series through fitting and summing up these items.

\subsection{MTGNN}

MTGNN $^{11}$ (Multivariate Time Graph Neural Networks) is a novel use of Graph learning layer and a Time convolution module is proposed to capture Time patterns through the modified one-dimensional convolution. The framework is shown in Figure 1 below:

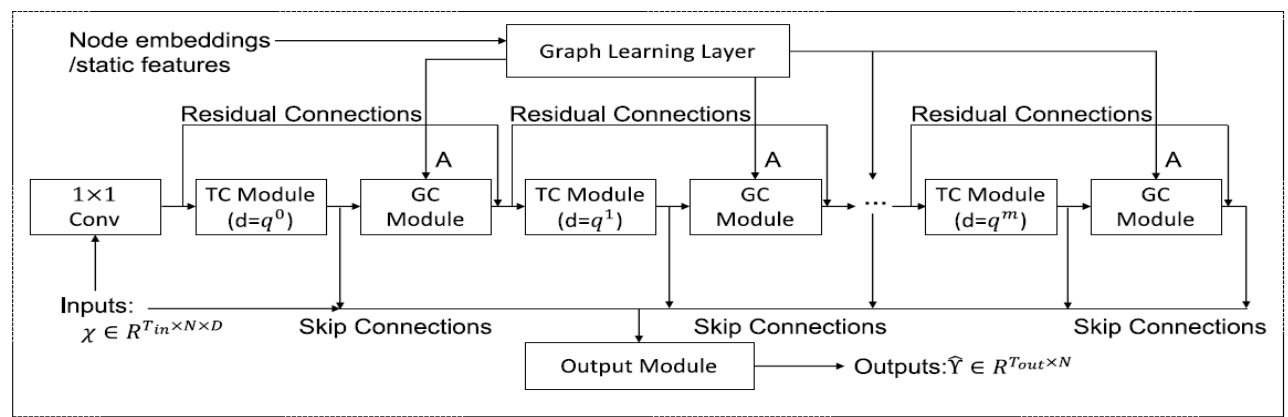

Fig 1. General framework of MTGNN

In multivariate time series prediction, the change of one node's condition may lead to the change of another node's condition. Therefore, the learning relationship should be one-way, as follows:

$$
\begin{gathered}
M_{1}=\tanh \left(\alpha E_{1} \theta_{1}\right) \\
M_{2}=\tanh \left(\alpha E_{2} \theta_{2}\right) \\
A=\operatorname{ReLU}\left(\tanh \left(\alpha\left(M_{1} M_{2}^{T}-M_{2} M_{1}^{T}\right)\right)\right) \\
\text { for } i=1,2, \ldots, N \\
i d x=\operatorname{argtopk}(A[i,:]) \\
A[i,-i d x]=0
\end{gathered}
$$

embedding, $\theta_{1}$ and $\theta_{2}$ are model parameters, $\alpha$ is the saturation rate of activation function, and argtopk(.) returns the subscript of the maximum value in the vector. Formula (10) is to calculate the asymmetric information of the adjacency matrix, in which the ReLU activation function can be used to regularize the adjacency matrix. Equations (12) and (13) dilute the adjacency matrix, which can reduce the computational cost and computational complexity of the convolutional network in the following figure. Figure convolution module and the structure of mixed jump propagation layer are shown in Figure 2:

Where $E_{1}$ and $E_{2}$ are randomly initialized node 

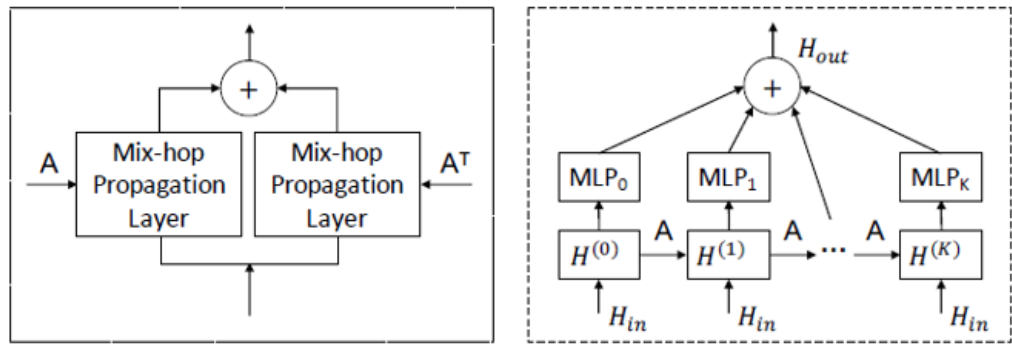

Fig 2. Graph Convolution Module and Mixed Jump Propagation Layer

The mixed jump propagation layer deals with the information flow on spatially related nodes, including two steps: the information propagation step and the information selection step. The steps of information transmission are as follows:

$$
H^{(k)}=\beta H_{\text {in }}+(1-\beta) A H^{(k-1)}
$$

Where $\beta$ is a hyperparameter that controls the rate at which the root node remains in its original state. Information selection steps are as follows:

$$
H_{\text {out }}=\sum_{i=0}^{K} H^{(k)} W^{(k)}
$$

The time convolution module uses multiple standard one-dimensional expansion convolution kernels to extract high-dimensional timing features, and its structure is shown in Figure 3:

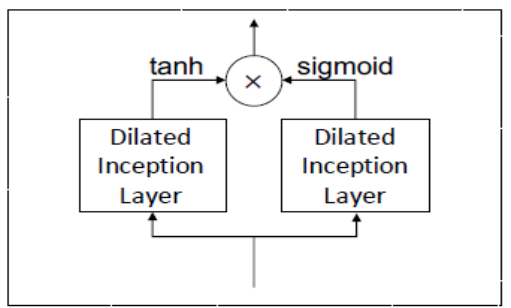

Fig 3. Time convolution module

The time convolution module captures the sequential mode of time series data through a one-dimensional convolution filter, which can simultaneously discover various range of time modes and process a long sequence of time convolution modules.

\section{Experiment}

\subsection{Model training}

There are mainly two types of data sets used in the experiment in this paper. One is the overnight offered rate data of Shibor on every working day, which includes 3,219 pieces of data on every working day from January 2007 to December 2019. There is also data on offered rate for each weekday of the 16 or 18 banks that make up Shibor. The experiment carried out in this paper is for the overnight variety because the overnight volume is the largest. The experiment of the first data set is mainly is to take a period of historical data to train and predict the data of a later period. The experiment of the second data set mainly uses the grey model $(1, N)$ to make the prediction. 1 represents the variables on the time line, and $\mathrm{N}$ represents the data of multiple dimensions. For example, when predicting 16 predicted values of the next working day of 16 quotation banks, $\mathrm{N}$ takes 16 .

\subsection{Prediction results and discussion}

The above two types of data were input into ARIMA, grey model, Prophet and MTGNN respectively to get the prediction results. In this paper, MAE and MSE are used to evaluate the performance of the above models, and the evaluation formula is as follows:

$$
\begin{gathered}
M A E=\frac{1}{n} \sum_{i=1}^{n}\left|y_{i}-\hat{y_{i}}\right| \\
M S E=\frac{1}{n} \sum_{i=1}^{n}\left(y_{i}-y_{i}^{\wedge}\right)^{2}
\end{gathered}
$$

Where $y_{i}$ and $y_{i}$ are the true value and the predicted value respectively; $n$ is the number of predicted samples. The smaller the evaluation values of MAE and MSE are, the higher the prediction accuracy of the model is. In order to compare and verify the performance of each model, four different models were used in this paper to calculate respectively based on the same training set and test set, and the experimental results obtained are shown in Table 1 below.

Table1. experimental results of each model

\begin{tabular}{|c|c|c|}
\hline Models & MAE & MSE \\
\hline ARIMA(1,2,0) & 0.2913 & 0.1973 \\
\hline Prophet & 0.2523 & 0.1569 \\
\hline Grey Model $(1,16)$ & 0.3301 & 0.2275 \\
\hline MTGNN & 0.1910 & 0.0825 \\
\hline
\end{tabular}

\section{Conclusion}

In this paper, four different models are used to predict the data of the interbank offered rate, and they are applied in the prediction of the actual interbank offered rate. This paper takes the data of each working day as the time scale of the interbank offered rate predict, and the research results are expected to provide help for bank management and interbank transaction decision making. In this paper, when predicting the interbank offered rate, it is divided into single-time dimension and multi-time dimension for comparison. However, considering that the two types of data do not consider the indicators of influencing factors, the accuracy of the model is relatively low. In the future work, macroeconomic factors and other features will be added to further predict and analyze the interbank offered rate, and these factors will be studied to obtain more accurate results. 


\section{Acknowledgments}

This research was funded by Chongqing Graduate Studen t Research Innovation Project: (CYS19257).

\section{References}

1. Esling, P. , \& Agon, C. . (2012). Time-series data mining. Acm Computing Surveys, 45(1), 1-34.

2. Fu, T. C. . (2011). A review on time series data mining. Engineering Applications of Artificial Intelligence, 24(1), 164-181.

3. Modarres, R. . (2007). Streamflow drought time series forecasting. Stochastic Environmental Research \& Risk Assessment, 21(3), 223-233.

4. Junior, P. R., Pamplona, E. D. O. , \& Fernando Luiz Riêra Salomon. (2014). Arima: an applied time series forecasting model for the bovespa stock index. Journal of Computer \& Communications, 5(21), 3383-3391.

5. Thompson, O. Y. . (2003). Short-term forecasting of crime. International Journal of Forecasting.

6. Nepal, B., Yamaha, M., Yokoe, A., \& Yamaji, T. (2020). Electricity load forecasting using clustering and ARIMA model for energy management in buildings. Japan Architectural Review, 3(1), 62-76.

7. Dash, P. K., Ramakrishna, G., Liew, A. C., \& Rahman, S. (1995). Fuzzy neural networks for time-series forecasting of electric load. IEE proceedingsgeneration, transmission and distribution, 142(5), 535-544.

8. Liu, X., Liu, H., Guo, Q., \& Zhang, C. (2019). Adaptive wavelet transform model for time series data prediction. Soft Computing, 1-8.

9. Alves, L. G., Ribeiro, H. V., \& Rodrigues, F. A. (2018). Crime prediction through urban metrics and statistical learning. Physica A: Statistical Mechanics and its Applications, 505, 435-443.

10. Taylor, Sean, J., Letham, \& Benjamin. (2018). Forecasting at scale. American Statistician, 72(1), 3745.

11. Wu, Z. , Pan, S. , Long, G. , Jiang, J. , Chang, X. , \& Zhang, C. . (2020). Connecting the dots: multivariate time series forecasting with graph neural networks. 